Meta

Journal des tradlucteurs

Translators' Journal

\title{
L'épidémiologie et les mots pour la dire
}

\section{Amal Jammal, Geneviève Loslier et Annie Gaillandre}

Volume 31, numéro 1, mars 1986

Traduction et terminologie médicale

Medical Translation and Terminology

URI : https://id.erudit.org/iderudit/003246ar

DOI : https://doi.org/10.7202/003246ar

Aller au sommaire du numéro

Éditeur(s)

Les Presses de l'Université de Montréal

ISSN

0026-0452 (imprimé)

1492-1421 (numérique)

Découvrir la revue

\section{Citer cet article}

Jammal, A., Loslier, G. \& Gaillandre, A. (1986). L'épidémiologie et les mots pour la dire. Meta, 31(1), 34-58. https://doi.org/10.7202/003246ar d'utilisation que vous pouvez consulter en ligne. 


\title{
L'ÉPIDÉMIOLOGIE ET LES MOTS POUR LE DIRE*
}

\author{
AMAL JAMMAL, GENEVIÈve LosliER, ANNIE GaILlANDRE
}

Comme dirait l'auteur des Lettres persanes : Comment peut-on être épidémiologiste ? Qu'est-ce qui, en effet, incite un médecin à s'intéresser à la maladie plutôt qu'au malade ? Car, à première vue, c'est ce que l'on pourrait penser puisque l'épidémiologie classique ${ }^{+}$peut se définir comme suit :

1. Étude des maladies épidémiques (infectieuses).

2. Dans un sens plus moderne, étude des maladies et de divers phénomènes biologiques ou sociaux du point de vue de leur fréquence, de leur distribution, et des facteurs susceptibles de les influencer (par ex. épidémiologie des maladies cardio-vasculaires, des accidents, des divorces, des suicides, etc. $)^{1}$.

Un grand absent : le malade. En apparence du moins, car on peut arguer, avec raison d'ailleurs, que ce dernier reste au centre des préoccupations de l'épidémiologiste et que la prévention, principal objectif de l'épidémiologie, a pour but ultime " un monde sans maladie et sans souffrance $»^{2}$.

Force est cependant de reconnaître que, si les objectifs du médecin clinicien et de l'épidémiologiste se rejoignent, leurs démarches sont sensiblement différentes. Le premier s'intéresse à l'individu : le malade en l'occurrence; le second, à une collectivité : la population dans laquelle la maladie se propage. Le clinicien cherche à poser le bon diagnostic, à déterminer le traitement le plus efficace et, par la suite, à en évaluer les résultats. L'épidémiologiste, pour sa part, tente de mesurer l'envergure et la fréquence de la manifestation de la maladie; cherche à en identifier les facteurs étiologiques et les facteurs de risque ; il s'efforce de mettre sur pied des programmes pour lutter contre elle, ou mieux encore, pour la prévenir. Il évalue ensuite l'efficacité de ces programmes pour, finalement, exercer une surveillance épidémiologique qui évitera la réapparition d'un autre phénomène de masse.

Comparativement à la médecine clinique, l'épidémiologie, dans le sens qu'on lui donne aujourd'hui, est une science relativement jeune, qui n'a pas fini de délimiter son champ d'étude puisque, nous l'avons vu plus haut, elle peut s'intéresser à l'étude d'un phénomène aussi bien social que pathologique. Et la sociologie le lui rend bien, qui puise abondamment dans le vocabulaire épidémiologique pour décrire certaines de ses réalités. C'est ainsi que des sociologues parleront d'épidémies de divorce, de facteurs de risque de mésadaptation sociale, etc. Par ailleurs, il suffit de penser à la complexité du principal objectif de l'épidémiologie, soit la prévention pour la santé et le bien-être des individus, pour comprendre aisément que cette discipline touche à la génétique, à la microbiologie, à l'immunologie mais aussi à la démographie, à l'écologie et, très souvent, à l'ubiquitaire statistique.

Rendu déjà complexe par la diversité des domaines qu'il dessert, et pour lesquels les mêmes termes ne désignent pas toujours les mêmes notions, le vocabulaire de l'épidémiologie connaît en outre les affres des choses qui commencent et que les anglophones 
réunissent sous l'expression pittoresque de teething troubles : tâtonnements pour trouver le mot juste, la locution la plus descriptive ; néologismes pas toujours concordants parce que, d'un auteur à l'autre, le trait descriptif le plus signifiant - celui qui refléterait le mieux la notion ou le concept — n'est pas forcément le même ; séries de termes assez voisins pour être source de confusion, mais pas assez équivalents pour être considérés comme de parfaits synonymes. Sans compter les démons familiers du traducteur : anglicismes, faux-amis, contresens, etc.

Pour dire l'épidémiologie et les difficultés de son vocabulaire, il nous a semblé intéressant de mettre nos pas dans ceux de l'épidémiologiste qui doit faire face à un phénomème de santé d'une envergure inhabituelle. Cependant, faute de temps et d'espace, nous avons choisi de privilégier plus particulièrement certains aspects de la démarche épidémiologique, ceux-là précisément qui nous permettraient d'étudier des termes controversés ou opaques, ou encore ceux dont les frontières sémantiques ne sont pas bien définies. De même, pour ne pas alourdir indûment le texte, nous avons évité de faire suivre ces termes - reconnaissables à leur impression en caractères gras - de leurs équivalents anglais. Ceux-ci figureront, sous forme d'un mini-glossaire, à la fin de l'article.

Avant de parler de la démarche de l'épidémiologiste, il serait peut-être bon de cerner le champ même de l'épidémiologie. Celui-ci, assez restreint au départ, n'a cessé de s'agrandir au fil des ans. Au début en effet, cette science s'intéressait uniquement aux épidémies, c'est-à-dire, selon l'acception originelle du terme, à l'augmentation inhabituelle de la fréquence des maladies infectieuses et contagieuses. Petit à petit, ce cadre devait s'élargir pour englober tour à tour les maladies transmissibles, qu'elles soient infectieuses ou non - et l'on notera que "transmissibles " a remplacé " contagieuses »-, puis les maladies non transmissibles. Ensuite, la notion de maladie elle-même éclate pour faire place à celle, plus large, de phénomène de santé. Ce dernier à son tour retiendra l'attention de l'épidémiologiste, non seulement lorsqu'il est de nature biologique,

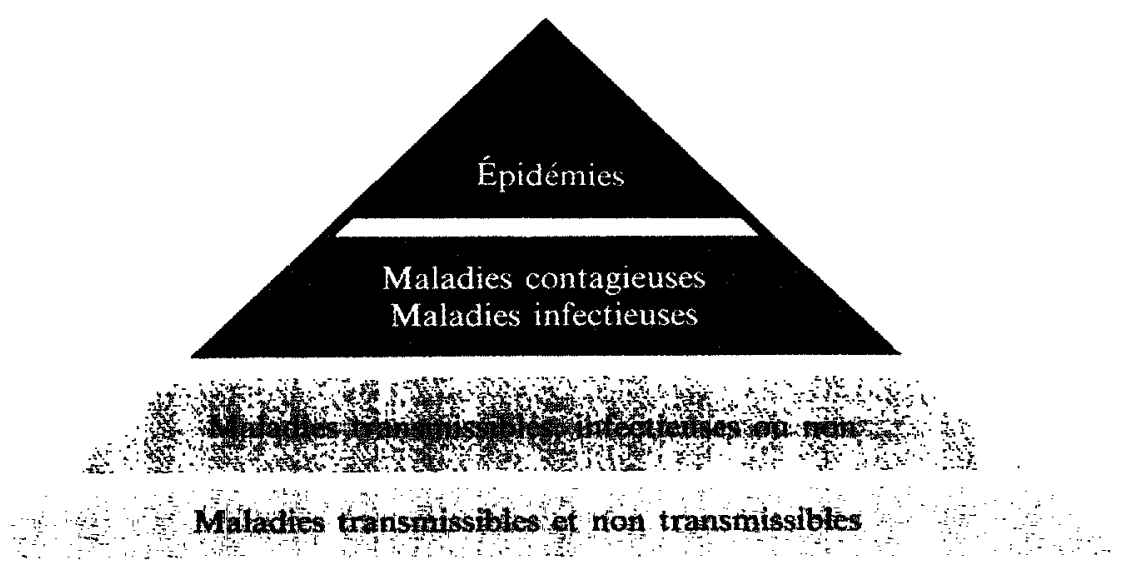

de nature biologique

Phénomènes de santé

d'origine sociale 
mais même lorsqu'il est d'origine sociale (suicides, divorces, accidents du travail, etc.). Par ailleurs, cette science ne se contentera pas d'une observation et d'une description du phénomène de santé, mais recherchera activement, par l'analyse et le raisonnement, à identifier l'ensemble des facteurs qui gouvernent l'apparition de ce phénomène, sa fréquence et sa distribution dans la population.

On pourrait représenter l'évolution du champ de l'épidémiologie par le schéma pyramidal présenté à la page précédente.

On est donc loin de l'étude des seules maladies infectieuses. Par ailleurs, l'épidémiologie est souvent qualifiée d'après l'orientation de sa démarche. Elle peut être une épidémiologie d'observation, comportant des études descriptives ou analytiques; ou une épidémiologie d'expérimentation, cherchant par des interventions appropriées à juguler les phénomènes pathologiques.

Revenons maintenant à notre épidémiologiste. En premier lieu, il cherche à mesurer l'ampleur du phénomène qui lui est signalé. Pour ce faire, il dispose de méthodes et d'outils de mesure particuliers. Ainsi, pour évaluer l'état de santé de la population dans laquelle le phénomène s'est manifesté, il utilise un certain nombre d'indicateurs de santé. À distinguer d'indice, terme qui semble réservé à des mesures composites combinant plusieurs indicateurs. Certains auteurs préfêrent d'ailleurs ne pas utiliser le mot indice dans ce contexte parce qu'il suggère un degré de précision qui n'est pas encore atteint en matière de santé ${ }^{3}$. Les principaux indicateurs sont donc, à part l'espérance de vie qui ne pose aucun problème terminologique, la morbidité, la mortalité, la létalité. Voilà un premier exemple de voisinage terminologique dangereux. Morbidité est sans équivoque. Par contre, mortalité et létalité risquent d'être pris pour synonymes. Or il n'en est rien, puisque les cas de mortalité sont les cas de décès dénombrés dans la population générale, alors que les cas de létalité sont les cas de décès dénombrés dans la population des malades.

Vient ensuite la confusion assez fréquente entre l'indicateur sous sa forme absolue et ce même indicateur sous sa forme relative. Ainsi, certains auteurs parleront indifféremment de mortalité et de taux de mortalité. Pourtant, à la lecture du Dictionnaire Flammarion, on serait porté à les différencier :

Mortalité : mot collectif par lequel on désigne la quantité d'hommes qui succombent à la même maladie (Littré). Taux de mortalité : Nombre total des décès annuels au sein d'une population calculé généralement sur la base de 100000 habitants 4 .

Donc, la mortalité serait exprimée par un nombre absolu ; le taux de mortalité par un rapport.

À signaler, en passant, que les notions de mortalité et de taux de mortalité, telles qu'elles sont définies par Flammarion, correspondent respectivement aux notions de mortalité pour une maladie donnée et de taux brut de mortalité décrites par Manuila et coll. $^{5}$

Ces derniers, pour leur part, présentent les deux notions - mortalité et taux de mortalité - comme des rapports, qui ne diffèrent que par la composition de leur dénominateur :

mortalité : fréquence des décès dans une période donnée par rapport à la population totale (les malades et les bien-portants) parmi laquelle ils surviennent. Le taux de mortalité exprime le nombre de décès dans une unité de population pour un temps déterminés. 
Comme on peut le voir, la mortalité, un sujet qui n'est réjouissant pour personne, l'est encore moins pour le terminologue. Et les difficultés ne s'arrêtent pas là ; qu'on en juge : pour désigner un certain indicateur, Mme Rumeau-Rouquette utilise l'expression part de la mortalité due à une cause. De prime abord, le syntagme semble incomplet, puisqu'on est tenté de se demander de quelle cause il s'agit. L'imprécision disparaîtrait si l'on ajoutait donnée, ce qui ferait part de la mortalité due à une cause donnée. Il est vrai que l'expression est rarement utilisée sous cette forme : on dira plutôt part de la mortalité due au cancer, aux pneumonies, etc. En second lieu, pour cette même notion définie ainsi :

Rapport du taux de mortalité pour une cause donnée au taux de mortalité générale 6

d'autres auteurs utilisent l'expression taux de mortalité proportionnel ${ }^{7}$ ou, ce qui peut sembler moins triste mais un peu bizarre, mortalité proportionnelle ${ }^{8}$. Selon le docteur Jenicek, l'expression taux de mortalité proportionnel, calquée sur l'anglais, proportionate mortality rate, serait à éviter. On est donc tenté de préférer la locution part de la mortalité due à une cause donnée, un peu longue mais plus explicite. Cette préférence serait encore renforcée par le fait que cette dernière expression se démarque, plus que la précédente, d'une expression voisine mais non synonyme, taux de mortalité par cause, qui désigne le

rapport du nombre de décès pour une cause donnée, une année donnée, à la population moyenne de l'année considérée?

Le dénominateur ici est la population moyenne, alors que dans la part de la mortalité due à une cause donnée, le dénominateur est le taux de mortalité générale.

Parmi les autres indicateurs, $i$ en est qui appelient certains commentaires ; ce sont prévalence, incidence et fréquence. Comme on le sait, la prévalence, notion statique, désigne le nombre de cas de maladie dénombrés au sein d'une population donnée, sans distinction entre les cas nouveaux et les cas anciens. On peut déterminer la prévalence à un moment précis; ce sera alors la prévalence à un moment donné ou prévalence ponctuelle, expression plus fidèlement descriptive que prévalence instantanée ${ }^{10}$ qui a une connotation de soudaineté, incomptatible avec un dénombrement de cas. Il est possible également de calculer la prévalence pour une période déterminée, et l'on parlera alors de prévalence au cours d'une période donnée ou de son synonyme prévalence de période $^{11}$. Ajoutons que ces expressions sont rarement employées telles quelles, mais plutôt d'une manière plus actualisée : prévalence au $1^{\mathrm{er}}$ janvier, prévalence en juin 1980, etc.

Quant à l'incidence, notion dynamique, elle ne s'applique qu'aux cas nouveaux ; elle traduit donc l'évolution d'un phénomène pendant une période donnée. Cette évolution sera mesurée par le taux de l'incidence, la vitesse de l'incidence, la densité de l'incidence.

Linguistiquement, incidence ne poserait aucun problème, n'était sa confusion avec fréquence, les deux termes étant souvent rendus en anglais par incidence. Sont-ils synonymes? Ni l'Organisation mondiale de la santé, ni les auteurs du Dictionnaire français de médecine et de biologie ne veulent se prononcer là-dessus; ils se contentent de faire suivre la déclaration de synonymie de l'avertissement " ambigu ". Même scénario pour le couple prévalence/fréquence. Mais encore? Faudrait-il bannir fréquence du vocabulaire médical ? Ne parler que d'incidence et de prévalence ? Ne faudrait-il pas plutôt considérer ce terme comme un générique, dont incidence et prévalence seraient des spécifiques ? En épidémiologie, la réponse est claire . le dénombrement peut inclure tous 
les cas (prévalence) ou les cas nouveaux (incidence). Mais qu'en est-il lorsqu'il s'agit, en médecine clinique par exemple, de la manifestation d'effets secondaires des médicaments ; lorsque la documentation anglaise mentionne the high incidence of side effects, sans précision chronologique? Comment le traducteur francophone déterminera-t-il s'il s'agit de prévalence ou d'incidence ? Le plus souvent, faute de mieux, il opte pour fréquence, terme décrié mais tellement pratique!

Rappelons encore que taux d'incidence et taux de prévalence ne sont pas synonymes d'incidence et de prévalence ; ceux-ci s'expriment par des nombres absolus, ceux-là par des rapports.

Au sujet de taux et de rapport, et de leur voisinage nocif avec ratio, proportion et pourcentage, il y aurait beaucoup à dire. En effet, si rapport et proportion sont presque synonymes, la proportion étant un rapport quantitatif (entre deux ou plusieurs choses) $^{12}$,

et s'il est évident que les deux peuvent s'exprimer en pourcentage, les termes rapport, taux et ratio ne sont pas équivalents. Le premier est un générique :

tout quotient de deux nombres l'un par l'autre ${ }^{13}$.

Les deux autres diffèrent par la composition de leur dénominateur. En effet, dans le cas de taux, le numérateur est compris dans le dénominateur : exemple :

\author{
10000 malades \\ population générale \\ (sujets sains et sujets malades)
}

Dans ratio par contre, le numérateur est différent du dénominateur : par exemple :

10000 malades

1000000 sujets sains

À noter cependant que Roland Pressat, dans son Dictionnaire de démographie, donne le sens de ratio à rapport, et définit ce dernier comme le résultat de la division de deux quantités entre lesquelles n'existe pas le type de correspondance permettant l'appellation de taux. Doit-on voir encore une fois l'influence de l'anglais qui appelle un rapport ratio?

À la lumière des données fournies par les indicateurs mentionnés plus haut, l'épidémiologiste pourra déterminer s'il s'agit d'une épidémie, d'une endémie ou d'une pandémie. La définition du terme épidémie varie d'un auteur à l'autre. Pour certaines, il s'agit du

développement subit et / de la/ propagation rapide d'une affection contagieuse dans une région où elle sévissait à l'état endémique, ou au sein d'une collectivité antérieurement indemne $e^{14}$.

Pour d'autres, c'est une :

apparition inhabituelle d'une maladie en tant que phénomène de groupe limité dans le temps et dans l'espace ${ }^{15}$. 
Deux nouveautés ici : la mention d'une maladie, sans épithète donc pas nécessairement contagieuse ou infectieuse, et la mention d'un phénomène de masse.

Enfin, à ces descripteurs, Manuila en ajoute d'autres :

Au sens moderne et par extension, multiplication considérable des cas de toute maladie (maladies transmissibles, carentielles, intoxications, etc.) ou de tout autre phénomène (accidents, suicides, etc. $)^{16}$.

Admission donc, dans la définition, des phénomènes non pathologiques.

L'endémie, pour sa part, est définie comme une

persistance habituelle, dans une région ou au sein d'une collectivité, d'une affection déterminée qui s'y manifeste de façon constante ou périodique ${ }^{17}$.

Ce qui la distingue donc de l'épidémie, c'est le descripteur "illimité dans le temps mais limité dans l'espace ${ }^{18}$ ").

La pandémie , enfin, est une

épidémie qui s'étend à presque tous les habitants d'une contrée et qui peut concerner une zone géographique très importante ${ }^{19}$.

Ici, c'est l'espace qui est illimité.

Il y en a même qui parlent d'anadémie pour désigner une

maladie non contagieuse qui attaque un grand nombre de personnes au même lieu ${ }^{20}$;

trait distinctif : l'absence de contagiosité.

Quand la propagation de la maladie a lieu chez les animaux, on parle d'épizootie, d'enzootie et de panzootie .

L'envergure du phénomène étant déterminée, l'épidémiologiste procédera à l'étude de ses particularités ou de ses caractéristiques : sa nature, les facteurs qui déterminent son apparition, sa fréquence et sa distribution. Il cherchera à cerner l'agent causal suspecté, les éléments qui favorisent sa multiplication et sa propagation, les risques et les facteurs de risque qui s'y rattachent, etc. Les démarches entreprises à cet effet peuvent porter plusieurs noms : étude, enquête, sondage, dépistage, expérimentation, essai, analyse. Ils sont tous intéressants pour le terminologue parce qu'ils appellent plusieurs remarques. En premier lieu, la confusion entre étude et enquête, assez fréquente dans les écrits épidémiologiques. Ces deux termes sont-ils équivalents au point d'être interchangeables? À l'évidence, non. C'est la distinction entre le tout et la partie, puisqu'une étude peut comporter une enquête mais aussi un essai et comprendre, en plus l'analyse des résultats et de leurs liens de causalité. Or, dans plusieurs écrits, quelquefois sous la plume d'un même auteur, voire dans le même paragraphe, on trouve indifféremment étude et enquête pour désigner la même notion.

Examinons maintenant la triade enquête, sondage, dépistage. Qu'est-ce qui les distingue, qu'est-ce qui les rapproche ? Les enquêtes épidémiologiques sont des

enquêtes pouvant utiliser des tests de dépistage qui n'ont pas principalement pour objet d'amener les malades à se faire soigner, mais de déterminer la prévalence, l'incidence et l'évolution des variables considérées, même si elles aboutissent naturellement à la découverte de cas qui seront mis en traitement ${ }^{21}$. 
Une petite parenthèse : les enquêtes épidémiologiques se distinguent par leur objectif premier - l'information - de la recherche des cas qui vise surtout à amener les malades à se faire soigner.

Par ailleurs, comme on a pu le constater, l'enquête peut prendre la forme de tests de dépistage, mais elle peut aussi comporter un sondage auprès d'un échantillon représentatif de la population. Il est donc clair que dépistage et sondage sont des formes d'enquête.

Cela dit, le dépistage lui-même pose des problèmes de terminologie. Il peut prendre la forme d'un dépistage de masse, d'un dépistage sélectif, d'un dépistage multiple ou d'un dépistage multiphasique. Là encore, à cause d'une ressemblance trompeuse entre ces deux dernières expressions, on peut penser qu'on est en face de deux synonymes. Le piège est d'autant plus traître que le dictionnaire unilingue anglais de Dorland ne semble pas faire de distinction entre les deux ${ }^{22}$. Et pourtant le dépistage multiphasique (peut-être synonyme de dépistage par examens successifs ${ }^{23}$ ?) comporte plusieurs méthodes ou plusieurs étapes de détection, chez un même sujet, d'une même maladie, alors que le dépistage multiple vise à déterminer la prévalence, chez des groupes nombreux, de deux ou plusieurs maladies. La différence, on le voit, est de taille.

Le sondage, pour sa part, fait appel au choix d'un échantillon. Ici, la confusion peut prendre plus d'une forme : confusion entre l'objet du choix, la méthode de ce choix et la méthode de l'enquête. L'expression qui met le plus clairement en évidence cette confusion est le sondage en grappes ${ }^{24}$ que d'aucuns appellent échantillonnage par grappes $^{25}$ ou échantillon en grappes ${ }^{26}$, ou encore, sondage par grappes ${ }^{27}$. Avouons-le, la récolte est bonne ! Que veut-on dire, lorsqu'on parle de grappes? Tout simplement un niveau d'agrégation, à mi-chemin entre l'individu et la population.

On ne dispose pas toujours, comme base de sondage, nous dit Mme Rumeau-Rouquette, d'une liste des unités. Mais on peut disposer d'une liste de groupes d'unités. Chacun de ces groupes, unité d'ordre supérieur appelée "grappe", contient une ou plusieurs unités qui nous intéressent ${ }^{28}$.

Mais alors, pourquoi pas groupe ? et peut-il y avoir une grappe avec une seule unité ? Cela dit, est-ce en ou par grappes? On opterait volontiers pour échantillon en grappes - puisqu'il s'agit de la forme que prend l'échantillon - et pour sondage par grappes, puisqu'il s'agit de la manière distributive de procéder.

Mentionnons également que l'enquête peut aussi être exhaustive si, au lieu d'être menée auprès d'un échantillon elle s'étend à toute la population. Elle peut par ailleurs être permanente ou ponctuelle.

Quant à l'expérimentation ou l'essai, ce sont aussi des étapes qui peuvent faire partie de l'étude, mais qui ne se confondent pas avec elle. L'expérimentation ou l'essai, par opposition à l'observation, sont des démarches de l'homme de science qui décide d'appliquer une mesure déterminée à lui-même, ou à des sujets ou groupes de sujets qu'il aura choisis.

L'évaluation des résultats se fait par rapport à une situation de référence. Exemple : on peut comparer une intervention à l'absence d'intervention, ou à une autre intervention du même type $^{29}$.

L'expérimentateur devra en général répartir les sujets choisis en deux groupes au moins. Cette répartition peut se faire par tirage au sort qu'on appelle encore répartition des sujets au hasard, distribution aléatoire ou allocation aléatoire. On voit que les termes ne manquent pas pour désigner la même notion. Pourquoi alors l'hybride randomisation, que l'échange du $\mathbf{z}$ contre l's ne suffit guère à franciser ? Le terme anglais, dérivé 
de random (hasard) se justifie pleinement mais, en français, où il n'a plus aucune assise, il passe mal.

L'essai, quant à lui, peut être à simple ou à double insu, ou encore en simple ou en double aveugle, selon que le sujet, ou le sujet et l'expérimentateur, ignorent qui reçoit le médicament et qui reçoit le placebo. On peut être surpris, à juste titre, par l'incongruité de l'expression en simple ou en double aveugle. En effet, si insu (sans que la chose soit sue de quelqu'un) exprime de façon elliptique mais acceptable la notion de non connaissance qu'implique l'essai à simple ou à double insu, l'expression à simple ou à double aveugle par contre semble entachée d'un jugement de valeur et d'une connotation péjorative, puisque aveugle, au sens figuré, nous dit le Robert, est celui " dont la raison, le jugement, est incapable de rien discerner. Loc. En aveugle : sans discernement, sans réflexion ". Ce n'est pas la connaissance ici qui fait défaut, mais bien le jugement, le discernement. Mais alors, pourquoi cette expression? Parce que l'anglais dit single ou double-blind study, évidemment.

Nous trouvons aussi l'expression essai contrôlé, calquée sur controlled trial. On peut se demander si, du moins dans le contexte épidémiologique, elle n'est pas redondante, car, par définition, tant l'essai que son équivalent trial, sont des expérimentations contrôlées. Qu'on en juge :

Trial : generally ... any controlled experiment with an outcome of an uncertain kind ${ }^{30}$.

Essai : expérimentation à visée thérapeutique, curative ou préventive où les sujets choisis sont divisés en groupes comparables à l'aide d'une méthode statistique ${ }^{31}$.

Il s'agit donc d'une démarche comportant un groupe témoin, par conséquent d'une démarche contrôlée.

À la lumière de ce qui précède - la distinction entre l'étude et ses composantes on se rend compte que certaines épithètes, telles que prospective, rétrospective, transversale, etc., dont on ne sait jamais s'il faut les accoler à l'étude, à l'enquête ou à l'analyse, qualifieraient mieux l'étude - plus globale - que l'une de ses étapes.

Il existe ainsi plusieurs types d'étude : l'étude cas-témoins, l'étude de cohortes, l'étude rétrospective, prospective, longitudinale, transversale, l'étude à simple insu, à double insu, l'étude descriptive, analytique, etc.

Certaines de ces expressions méritent de figurer au palmarès des casse-tête terminologiques. Â la place d'honneur : l'étude cas-témoins. Dira-t-on jamais assez le danger des calques aveugles de l'anglais ? Dans un article publié dans le Concours médical du 8 octobre 1983, sous la rubrique "Entre deux mots", le docteur J.C. Sournia cite le cas de borderline qui, dans le contexte particulier des tumeurs osseuses, signifiait à la limite (de l'état de malignité). Le mot fut transposé littéralement et traduit par ligne de bordure, par des chirurgiens français, avec le sens de "tumeur de la ligne de bordure ", c'est-àdire une tumeur prenant naissance sur la ligne qui sépare le périoste et le tissu osseux !

Dans le cas qui nous occupe, le coupable est case-control study, transposé en français, mot pour mot, trait d'union compris, pour donner étude cas-témoins. Examinons d'abord le mot témoin. Il fait partie, tant dans la langue courante que dans la langue médicale, de ce que l'on appelle une expression binominale qui s'écrit avec ou sans trait d'union, si l'on en croit le Dict. Robert, et dont le deuxième élément est un attribut du premier : personne ressource, appartement-modèle, lampe témoin et, plus particulièrement dans le contexte médical, groupe témoin, ou groupe-témoin. Dans cette dernière expression donc, le groupe est témoin. On pourrait par conséquent croire que, dans étude cas-témoins, il s'agit de cas qui sont témoins. Or, il n'en est rien, l'étude castémoins étant une étude dans laquelle " un groupe de personnes atteintes d'une certaine maladie est comparé à un groupe de témoins choisi de façon appropriée ${ }^{32}$. Il s'agit 
donc d'une étude comportant des cas et des témoins, et non de cas qui sont témoins. Pour illustrer cette ambiguïté, citons la phrase entendue sur les ondes de Radio-Canada, le 14 décembre 1983, au sujet d'une étude commandée par la Cie Alcan, relative à la fréquence du cancer de la vessie parmi les travailleurs des alumineries québécoises :

Selon les résultats de l'enquête menée auprès de 85 cas témoins par l'École de santé au travail de l'Université McGill, les travailleurs ouvrant dans les salles de cuves Soderberg ... sont particulièrement vulnérables face au cancer de la vessie.

Oui mais, nous dira-t-on, il s'agit de non-initiés ! Certes, mais que fait-on alors de ce paragraphe, lu dans l'Épidémiologie :

L'étude des cas témoins consiste à interroger les malades avérés sur leur comportement visà-vis du facteur de risque suspecté33.

et plus loin

On peut étudier les faits rétrospectivement : à partir de dossiers pris dans des archives, hospitalières en particulier; il ne faut pas confondre cette méthode avec l'étude des cas témoins $^{34}$.

On est autorisé à croire que, pour ces auteurs, les cas sont témoins ; à preuve : l'article défini devant le syntagme binominal.

L'expression pèche par un deuxième aspect : Elle compare des éléments non comparables. En principe, on oppose des personnes malades à des personnes témoins, mais si l'on écrit cas-témoins, c'est que l'on fait l'équation suivante :

$$
\text { cas }=\text { malades. }
$$

Or si, éthiquement, la chose en soi est difficilement admissible, mathématiquement, elle est tout simplement fausse. En effet, un malade peut présenter plus d'un cas de maladie. Il peut présenter plusieurs cas de cancer par exemple (cancer de la gorge, de la vessie, etc.). S'il fait dans la même année plusieurs rechutes d'une même maladie, il représentera autant de cas de cette maladie.

Toutefois, l'usage tout-puissant ayant presque consacré l'expression étude castémoins, on pourrait du moins lever partiellement l'ambiguïté en remplaçant le trait d'union par une barre oblique, symbolisant l'opposition, et écrire : étude cas/témoins.

Mais nous n'en avons pas fini avec ce type d'étude. En effet, pour certains, elle aurait comme synonyme étude rétrospective. Il est vrai qu'une étude cas-témoins suppose un retour dans le passé pour remonter le cours des événements. Autrement dit, elle est par définition rétrospective. Mais l'inverse est-il toujours vrai ? Et ne peut-on envisager une étude rétrospective qui ne comporterait au départ qu'un groupe présentant l'élément à l'étude ? Le qualificatif rétrospective, comme prospective ou longitudinale d'ailleurs, indique seulement l'axe temporel de l'étude et non la méthode qui y est utilisée. Même réserve à l'égard de la relation synonymique entre étude de cohortes et étude prospective. L'étude de cohortes est une

étude d'observation /qui/ ... habituellement ... /consiste/ à choisir une population exposée au facteur étudié et une population témoin non exposée, mais aussi proche que possible de la population exposée, au regard de facteurs concomitants connus ou supposés. Ensuite, on suivra dans le temps les deux groupes afin d'évaluer la survenue des nouveaux cas de maladies (incidence) ou éventuellement d'autres événements (décès) ${ }^{35}$.

Autrement dit, si dans l'étude cas-témoins on connaît les malades et on recherche les facteurs étiologiques, dans l'étude de cohortes, on connaît les facteurs de risque et l'on 
observe si, dans le futur, ils peuvent produire la maladie. Ici encore, la direction temporelle de l'étude n'est que l'un de ses descripteurs; il ne suffit pas à établir sa synonymie avec étude prospective. En effet, celle-ci est simplement une

étude orientée vers le rassemblement futur des données ${ }^{36}$

permettant

de suivre un groupe de sujets (ou plus généralement d'unités statistiques), afin d'étudier les phénomènes qui les affectent au cours du temps ${ }^{37}$.

Quant au terme cohorte, il pose aussi quelques problèmes. En fait, il désigne

un ensemble d'individus ayant vécu un événement semblable pendant la même période ${ }^{38,39}$. Il peut s'agir des personnes nées au cours d'une même année (génération), mais aussi de personnes hospitalisées à partir d'une même date ou ayant commencé une maladie au cours d'une même période ${ }^{40}$.

Selon le Dictionnaire Flammarion, le terme aurait un champ sémantique plus étroit et désignerait un

échantillon de population étudiée formé de sujets nés pendant une même période déterminée ${ }^{41}$.

À notre avis, corroboré par la Gazette officielle du Québec du 25 août 1984 précisant qu'il ne faut pas confondre cohorte et génération, la définition de Flammarion s'appliquerait plutôt à cohorte de naissance ou génération.

Quant au Dictionnaire Manuila ${ }^{42}$, il donne comme synonyme de cohorte, le terme promotion, différant en cela de Roland Pressat, qui limite l'usage de promotion à promotion de mariages ou cohortes de mariages ${ }^{43}$. Il est vrai que maintenant nous sommes en démographie. Ailleurs, la Gazette officielle nous dit encore de ne pas confondre cohorte et promotion ${ }^{44}$. Sauf que maintenant nous sommes en statistiques scolaires !

Ces distinctions, qui varient d'un domaine à l'autre, ne facilitent guère la tâche au terminologue.

Nous laissons là le chapitre des études pour aborder celui de leurs résultats. L'étude peut en effet révéler à l'épidémiologiste l'éclosion d'une maladie épidémique. Celle-ci peut être infectieuse, contagieuse ou transmissible. Qu'est-ce qui distingue les uns des autres ces termes voisins mais non synonymes?

Une maladie infectieuse est

toute maladie due à une infection se manifestant par des symptômes ou des signes cliniques. La plupart ... se transmettent directement ou indirectement d'une personne à une autre (maladies transmissibles), certaines d'entre elles par contagion (maladies contagieuses), mais d'autres, telles que la septicémie, ne sont pas transmissibles ${ }^{45}$.

Donc, toutes les maladies infectieuses ne sont ni nécessairement contagieuses ni forcément transmissibles. Autrement dit, le descripteur déterminant est la présence d'une infection qui n'implique pas nécessairement la contagion.

Quant à la distinction entre la maladie contagieuse et la maladie transmissible, elle est rendue plus confuse par la définition que donne Manuila de la maladie conta- 
gieuse :

Toute maladie infectieuse qui peut être transmise d'une personne à une autre, soit par contact direct (contagion directe), soit par l'intermédiaire d'une personne, d'un objet ou d'une substance contaminés par le sujet infecté (contagion indirecte) ${ }^{46}$.

Mais alors, quelle différence avec la maladie transmissible, que le même auteur définit ainsi :

Maladie attribuable à un agent infectieux spécifique ou à ses produits toxiques et qui survient par la transmission de cet agent ou de ses produits d'un réservoir à un hôte réceptif, directement par une personne ou un animal infecté, ou indirectement par l'entremise d'un hôte animal ou végétal intermédiaire, d'un vecteur ou du milieu extérieur inanimé47.

Les deux peuvent être transmises directement ou indirectement. Un seul trait distinctif de la maladie transmissible toutefois : la présence de l'hôte animal intermédiaire ou vecteur comme agent de transmission.

Et qu'est-ce qui distingue l'infection de l'infestation? La contagion de la contamination? L'infection de la contamination?

Selon le Dictionnaire Flammarion infection et infestation étaient considérés autrefois comme analogues — on ne sait si, par là, l'auteur signifie qu'ils étaient considérés comme synonymes. L'auteur ajoute :

ce dernier mot (infection) ayant été longtemps réservé aux virus et bactéries ${ }^{48}$.

Or, toujours selon le même auteur, la codification actuelle veut qu'on emploie le terme infection pour les virus, les bactéries et les parasites et que l'on réserve le terme d'infestation aux arthropodes ectoparasites (c'est-à-dire proliférant à la surface du corps). Le Dictionnaire français de médecine et de biologie de Manuila corrobore cette opinion mais mentionne une autre tendance qui veut qu'on définisse l'infestation comme une

fixation d'ectoparasites sur un hôte ou pénétration dans son organisme d'un parasite, visible, au moins à l'état adulte, sans emploi du microscope ${ }^{49}$.

En d'autres termes, pour certains, le trait distinctif est la taille de l'envahisseur; pour d'autres, le lieu de l'invasion. L'imprécision demeure. Il existe aussi le terme parasitose que désigne les maladies infectieuses « déterminées par la présence d'organismes vivants qui n’appartiennent pas à la catégorie des bactéries ni à celle des virus ${ }^{50}$.

Signalons, par ailleurs, que l'individu agressé peut souffrir également d'une surinfection, c'est-à-dire d'une infection (généralement nosocomiale) qui s'ajoute à l'infection originale. On ne devrait pas, selon Manuila, désigner cette notion par l'expression abusive d'infection croisée - calquée sur l'anglais cross infection - puisqu'il ne s'agit pas dans ce cas d'un croisement (analogue à celui de l'immunité croisée par exemple). À noter cependant que la définition du terme anglais cross infection permet de préciser que la surinfection provient de la contamination du malade par un germe qui lui a été transmis directement ou indirectement par un autre malade. Elle se démarque donc de superinfection où la surinfection est causée par un germe provenant de l'organisme même qui en sera la victime.

Quant à contamination et contagion, Manuila les donne comme synonymes, en avertissant le lecteur toutefois qu'il s'agit d'un emploi abusif ${ }^{51}$. Et pour cause : la contamination peut s'appliquer tant à l'animé qu'à l'inanimé (un objet peut être contaminé), alors que la contagion ne s'applique qu'à l'animé. 
Pour ce qui est du couple contamination/infection, là encore on peut parler de pseudo-synonymie puisque la contamination, même celle d'un être vivant par un germe, n'est pas nécessairement suivie d'une infection. Elle ne le sera que si l'organisme contaminé est favorable au développement des micro-organismes envahisseurs et même, pour certains $^{52}$, seulement si la présence de l'entité étrangère provoque des troubles pathologiques dans l'organisme de l'hôte.

Et puisque l'hôte entre en scène, il est temps d'en décrire les traits :

Être vivant hébergeant un animal ou un végétal qui se nourrit à ses dépens (et que l'on appelle, pour cette raison, parasite) ${ }^{53}$.

L'hôte peut être définitif, intermédiaire - actif ou passif -, hôte réceptif ou encore hôte de réenkystement. Il est définitif si le parasite y atteint la maturité sexuelle qui lui permet de donner naissance à un nouveau cycle ; synonyme : hôte primaire. Il est intermédiaire s'il héberge la forme larvaire du parasite, qui doit y séjourner un certain temps avant d'atteindre la forme infestante pour l'hôte définitif. Il sera intermédiaire actif s'il assure la transmission de l'agent pathogène par une action qui lui est propre (injection de salive, défécation, etc.) ; passif, s'il est incapable d'assurer par ses propres moyens la transmission de l'agent pathogène qu'il héberge (transmission qui est alors assurée par l'écrasement ou l'ingestion de l'hôte). Il est hôte de réenkystement s'il s'agit d'un organisme au sein duquel la forme larvaire du parasite ne trouve pas de conditions favorables à son développement et à son évolution, et chez lequel elle s'enkyste ; synonymes : hôte d'attente, hôte paraténique ${ }^{54}$.

De l'hôte, nous passons à l'agent de transmission. Est-ce un vecteur ? un véhicule ? un agent disséminateur ? S'il est vrai que ces acteurs participent tous trois à la propagation de la maladie, ils ne sont pas pour autant synonymes. En effet, un vecteur est :

en principe, tout animal capable de transmettre un agent infectieux d'un hôte à un autre. En fait, le sens de ce mot est restreint aux organismes appartenant à un embranchement différent de celui auquel appartient l'organisme infectés5.

Exemple : anophèles pour le paludisme, poux pour le typhus. $\grave{A}$ ne pas confondre avec agent disséminateur qui ne transmet pas directement l'agent infectieux d'un hôte à un autre. Exemple d'un agent disséminateur : la mouche qui transporte le poliovirus, à partir des excréments, sur les aliments.

À ne pas confondre également avec véhicule, terme qui ne s'applique qu'à l'objet ou à la substance à l'aide desquels s'est opérée la transmission de l'agent causal.

Et nous voilà face au premier responsable, l'agent causal ou agent étiologique. L'étude cherchera à déterminer sa virulence, son pouvoir pathogène, ou pathogénicité, et son pouvoir envahissant. Là encore, un voisinage dangereux.

La virulence est la capacité d'un germe infectieux à se multiplier dans l'organisme infecté et d'y provoquer des troubles morbides. Elle constitue donc une des modalités du pouvoir pathogène et s'exprime par le rapport du nombre de décès ou de cas graves sur le nombre de personnes infectées et malades.

Le pouvoir pathogène, pour sa part, est la capacité d'un agent infectieux de causer la maladie chez un individu ; il s'exprime par le rapport du nombre de personnes infectées et malades sur le nombre de personnes infectées. Donc, tant les numérateurs que les dénominateurs sont différents. 
Quant au pouvoir envahissant, c'est l'aptitude d'un germe à se propager, après pénétration dans l'organisme, à travers les différents organes, tissus et systèmes anatomophysiologiques ${ }^{56}$. Il se distingue donc nettement de la virulence et du pouvoir pathogène par l'absence du descripteur " provocations de troubles morbides". A titre d'exemple : certains germes comme Clostridium tetani sont très virulents mais dotés d'un faible pouvoir envahissant ; d'autres, comme Salmonella, sont moins virulents mais possèdent un pouvoir envahissant considérable.

Une fois l'organisme infecté, il peut connaître une période d'incubation ou encore une période de latence, termes qui ne sont pas de parfaits synonymes. La période d'incubation désigne en général l'intervalle qui sépare l'infection de l'apparition du premier signe ou du premier symptôme de la maladie. La période de latence, quant à elle, n'est pas une notion réservée aux maladies infectieuses ; elle peut s'appliquer aussi bien aux phénomènes allergiques qu'à certaines maladies héréditaires ou neuropsychiatriques. La prépatence désigne, pour sa part, la période qui s'étend du jour de l'infection par un organisme au jour où apparaissent des formes ou des manifestations de cet organisme, décelables par les méthodes biologiques.

Voilà décrits l'agresseur et ses caractéristiques. L'étude peut alors tenter de déterminer les facteurs qui en influencent l'apparition, la fréquence, la distribution et l'évolution. Elle cherchera à évaluer le risque, à déterminer les facteurs de ou du risque, légèrement distincts des marqueurs du risque ; à reconnaître la population à risque élevé ; à mesurer la fraction étiologique du risque, à ne pas confondre avec le risque attribuable ou avec le risque lié à un facteur donné, etc.

Nous voyons d'emblée que gravitent autour du risque plusieurs expressions qui ne sont pas toutes transparentes. Qu'est-ce que le risque ? "Péril dans lequel entre l'idée de hasard ", nous dit le Littré.

Probabilité de survenue d'un événement. S'il s'agit d'une maladie, une estimation en est donnée par la fréquence de la maladie au sein de la population considérée

nous dit une éminente épidémiologiste ${ }^{57}$. C'est donc essentiellement une probabilité quantifiable.

Et le facteur de risque ou du risque ? C'est l'

attribut ou caractéristique physiologique, pathologique, culturel ou lié à l'environnement, entraînant, pour l'individu chez lequel on le détecte, une probabilité plus élevée d'être frappé par une affection donnée (par exemple la consommation de cigarettes est un facteur de risque vis-à-vis du cancer du poumon) ${ }^{58}$.

Le risque exprime donc une probabilité, le facteur de risque, tout critère dont elle dépend $^{59}$. Certains auteurs ${ }^{60}$, par ailleurs, font la distinction entre facteur de risque et marqueur du risque selon que l'élément qui influe sur le risque est un caractère inhérent à l'individu, comme l'âge, le sexe (marqueurs du risque) ou un caractère acquis et modifiable comme l'usage de la cigarette ou l'obésité (facteurs du risque).

D'autre part, le risque peut être absolu, relatif, mais il peut également être attribuable. Oui, attribuable tout court, l'expression étant sans doute calquée sur l'anglais attributable risk. La question qui vient aux lèvres, tant anglaises que françaises, est bien entendu attribuable à qui ou à quoi ? Flammarion nous donne pour risque attribuable :

Différence entre la probabilité de contracter une maladie quand on est exposé et la probabilité quand on est (sic) pas exposét1. 
Même définition chez Monnier et coll ${ }^{62}$. L'ellipse, aussi bizarre soit-elle, semble admise dans la langue épidémiologique.

Mais là ne s'arrête pas l'anomalie. Pour certains, en effet, le risque attribuable est une différence arithmétique : c'est ce qui reste si on soustrait le risque chez les nonexposés du risque chez les sujets exposés $63,64,65$. Pour d'autres, l'expression serait synonyme de proportion de cas dus au facteur ${ }^{66}$ (lequel ? toujours l'ellipse !). Pourtant elle désigne la différence entre la fréquence des cas dans le groupe exposé et la fréquence des cas dans le groupe non exposé, rapportée à la fréquence des cas dans les deux groupes. Cette définition se rapproche par ailleurs de celle que les épidémiologistes Levin et $\mathrm{Li}$ lienfeld donnent de attributable risk ${ }^{67}$.

La fraction étiologique du risque, selon le docteur Jenicek, serait le risque attribuable exprimé en proportion du risque total dans un groupe ${ }^{68}$, définition très proche de proportion de cas dus au facteur, mais différents de celle de risque attribuable tout court. Voici donc deux expressions égales à une troisième mais qui ne sont pas égales entre elles.

Et la population à risque, calquée une fois de plus sur l'anglais population at risk ? Est-elle une population exposée au risque ? une population vulnérable au risque ? une population représentant un risque pour d'autres?

Une population peut être exposée à un risque donné, nous dit un épidémiologiste, du fait de son exposition à un facteur donné relié ou non, sur le plan étiologique, à la maladie ... sans tenir compte de l'aptitude des sujets à contracter la maladie ${ }^{69}$. Serait-ce cette aptitude qui en ferait une population vulnérable ? Et cette expression serait-elle synonyme de population prédisposée? Autant de questions qui n'ont pas encore reçu de réponses unanimes.

Par ailleurs, que doit-on faire du syntagme, bien ancré dans la langue épidémiologique, population à haut risque ou à risque élevé. Il serait difficilement délogeable par population fortement exposée ou population très vulnérable.

Après avoir identifié les caractéristiques de la maladie, le risque et ses facteurs, ainsi que les populations qui y sont exposées, l'épidémiologiste s'attachera à l'éradication de la maladie, soit à sa suppression propre, soit à la suppression de son vecteur. Pour ce faire, il recommandera les mesures nécessaires à la lutte contre la maladie. À noter que cette expression est moins triomphaliste, mais peut-être plus réaliste, que sa contrepartie anglaise control of the disease ou disease control. Que l'on nous permette ici de crier au piège à qui serait tenté par l'anglicisme "contrôle de la maladie ", sous prétexte que le mot a fait son chemin dans la langue française, avec l'acception de maîtrise, et qu'il est en passe d'être consacré par l'usage. Une des tâches de l'épidémiologiste, et non la moindre, est justement la surveillance épidémiologique, très proche du sens orthodoxe de contrôle. Que l'on parle de contrôle de la maladie et l'interlocuteur ne saura plus s'il s'agit de la surveillance de la maladie ou de la maîtrise de la maladie.

L'intervention de l'épidémiologiste peut donc prendre la forme d'un programme thérapeutique pour endiguer l'avance d'une épidémie, mais elle peut prendre aussi la forme d'un programme de prévention. Or, toute intervention implique des coûts, parfois considérables. Il faut donc évaluer l'utilité, l'efficacité, le rendement ou efficience de ces programmes. Les termes rendement et efficacité, très voisins, ne sont pas synonymes. L'efficacité mesure jusqu'à quel point un programme a atteint ses objectifs ; l'anglais. rend cette notion par effectiveness. Le rendement mesure les résultats obtenus, par rapport aux moyens utilisés (coûts, ressources, temps) ; à ce titre, il serait synonyme d'efficience, et il est rendu en anglais par efficiency. 
Mais il ne s'agit pas là des seuls critères d'évaluation de l'intervention. On peut évaluer cette dernière par les méfaits potentiels de la maladie qu'elle permettrait d'éviter. Pour cela, l'épidémiologiste dispose d'un certain nombre de mesures : ainsi l'indicateur faussement élégiaque que sont les années potentielles de vie perdues (abréviation : APVP), concept représentant le nombre d'années qu'un sujet, mort prématurément c'est-à-dire, selon un choix arbitraire, avant l'âge de 70 ans - n'a pas vécues. Cet indicateur permet de comparer l'importance relative de diverses causes de décès dans une population donnée, pour une période donnée.

Vient ensuite l'indicateur années-personnes en bonne santé, à la syntaxe boîteuse, qui mesure le cumul des années gagnées grâce à un programme de santé par les personnes constituant la population cible de ce programme, dans le cas d'une maladie dont on peut attendre une guérison totale. (Exemple : la tuberculose.) Il se fonde sur la mesure de la fréquence de la maladie avant et après l'application du programme.

Vient enfin l'indicateur années de validité. Cet indicateur s'applique dans le cas d'une maladie pouvant laisser des séquelles permanentes même si elle est traitée mais qui, en l'absence du programme, aurait de plus laissé l'individu invalide (exemple : certaines maladies arthritiques). On peut estimer ainsi le "gain sanitaire " attendu d'un programme de santé, par la mesure de la fréquence de l'invalidité dans la population, avec et sans l'application du programme. Nous voici devant le mot invalidité et devant la série de termes qu'il évoque : infirmité, incapacité, déficience, handicap. Ces mots sont-ils des synonymes? Sinon, qu'est-ce qui les distingue ? Nous devons avouer que la différence est très ténue. Nous serions tentées de l'appeler une différence "d'éclairage sémantique ».

On pourrait estimer que déficience et infirmité sont des termes génériques englobant toute perturbation ou limitation d'une fonction - y compris les fonctions mentales - qui entraînent une perturbation du fonctionnement normal de l'organisme dans son ensemble. Les trois autres pourraient être des spécifiques. Encore une fois, la différence entre eux est subtile.

L'incapacité serait :

/'/état d'une personne qui, à la suite d'un accident ou d'une maladie, a perdu la plénitude de ses possibilités professionnelles pour des raisons physiques ou mentales ${ }^{70}$.

Ressort ici le descripteur "entrave au travail professionnel».

Le handicap, dans un sens restreint, est chez l'individu qui en souffre un :

désavantage résultant d'une déficience ou d'une incapacité, qui gêne ou limite le sujet dans l'accomplissement de son rôle social'1!

Élargissement de la notion précédente avec l'inclusion du descripteur " entrave au rôle social $\%$.

L'invalidité recouvre à la fois les notions de handicap et d'incapacité, mais semble ressortir principalement au domaine des lois sociales (assurances, indemnités, etc.).

Ayant fait tout ce qu'il pouvait pour arrêter l'avance de l'épidémie, l'épidémiologiste devra finalement penser à la surveillance épidémiologique que nous avons évoquée plus haut et qui aurait pour synonyme, monitoring. Ce terme, moins français, peut être de plus source de confusion ; il existe en effet un mot voisin, monitorage, qui recouvre une tout autre notion puisqu'il désigne la surveillance d'un sujet utilisant un moniteur (cardiaque par exemple).

Nous avons ainsi évoqué, sans prétendre les résoudre, certains problèmes du vocabulaire de cette discipline qui, de par son jeune âge et surtout de par sa situation au car- 
refour de plusieurs domaines, n'a pas fini en quelque sorte de «chercher ses mots». Nous espérons par ailleurs, à l'occasion de ce survol très rapide de l'épidémiologie, avoir répondu implicitement à la question du début, et démontré que le souci premier de l'épidémiologiste, loin d'être une recherche désincarnée des particularités d'une maladie, est essentiellement le mieux-être de la population. Pour terminer, que l'on nous permette de citer ces phrases par lesquelles le docteur Jenicek clôt son Introduction à l'épidémiologie :

L'utilité du travail épidémiologique ne se manifeste souvent qu'au moment où on cesse de le faire (surtout dans le cas de la surveillance épidémiologique). Son succès n'est pas aussi flagrant aux yeux de la population que la guérison clinique d'un patient. Il faut parfois faire de l'épidémiologie avec l'esprit des premiers chrétiens. ments?

Comment s'empêcher, lorsqu'on est traducteur, de faire certains rapproche-

\section{LEXIQUE FRANÇAIS-ANGLAIS}

agent causal

syn. : agent étiologique

agent disséminateur

agent étiologique, syn. de agent causal

allocation alléatoire, syn. de tirage au sort

anadémie

analyse

années potentielles de vie perdues (abrév.

$$
\text { APVD) }
$$

années-personnes en bonne santé

années de validité

APYP (abrév. de années potentielles de vie perdues)

cas

cohorte

cohorte de mariage

syn : promotion

cohorte de naissance

syn. : génération

contagion

contagion directe

contagion indirecte

contamination

déficience

densité de l'incidence

dépistage

dépistage de masse

dépistage multiphasique, syn. de dépistage par examens successifs?

dépistage multiple

dépistage par examens successifs

syn. : dépistage multiphasique?

dépistage sélectif

dépistage systématique

distribution aléatoire, syn. de tirage au sort causal agent; agent; etiological agent

disseminating agent

sans équivalent

analysis

potential years of life lost

person-years of health

person-years without incapacity?

case

cohort

birth cohort

contagion

direct contagion; immediate contagion

mediate contagion

contamination

impairment

incidence density; person-time incidence rate

instantaneous incidence rate; hazard rate

screening

mass screening

multiple screening

multi-step screening

selective screening

sans équivalent 
échantillon

échantillon en grappes

échantillonnage

échantillonnage en grappes

efficacité

efficience, syn. de rendement

endémie

enquête

enquête épidémiologique

enquête exhaustive

enquête permanente

enquête ponctuelle syn. : enquête transversale enquête par sondage

syn. : sondage

enquête transversale, syn. de enquête ponctuelle

enzootie

épidémie

épidémiologie d'expérimentation

épidémiologie d'observation

épizootie

éradication (d'une maladie)

espérance de vie

essai

essai comparatif

syn. : essai "contrôlé "

essai à double insu

syn. : essai à double aveugle ;

essai en double anonymat

essai à l'insu

essai à simple insu

syn. : essai à simple aveugle;

étude

essai en simple anonymat

étude analytique

étude cas / témoins *

étude comparative des cohortes, syn. de étude de cohortes

étude de cohortes

syn. : étude comparative des cohortes étude descriptive

syn. : étude de prévalence

étude longitudinale

étude de prévalence, syn. $d$ 'étude descriptive

étude prospective

étude rétrospective

étude transversale

expérimentateur

expérimentation

facteur de risque; facteur du risque

facteur étiologique

fraction étiologique du risque

syn. : proportion de cas dus au facteur; fraction attribuable

fréquence (de la maladie)

fréquence globale, syn. peu usité de prévalence sample

cluster sample?

sampling

cluster sampling?

effectiveness

endemic ; endemia ; endemy

survey

epidemiological survey

comprehensive survey?

permanent survey

cross-sectional survey

sample survey

enzootic; enzootia; enzooty

epidemic

experimental epidemiology

observational epidemiology

epizootic ; epizootia ; epizooty

eradication (of disease)

life expectancy; life expectation

trial

controlled trial; controlled intervention study

double blind trial

blind trial

single blind trial

study

analytical study

case control study

cohort study; incidence study

descriptive study; prevalence study

longitudinal study

prospective study

retrospective study

cross-sectional study

experimenter

experimentation; intervention study

risk factor

etiological factor

etiological fraction; attributable risk percent population attributable risk; population attributable risk proportion

frequency (of disease) 
génération, syn. de

cohorte de naissance

groupe témoin

handicap

hôte

hôte alternant, syn. de hôte intermédiaire

hôte d'attente, syn. de hôte paraténique

hôte définitif syn. : hôte primaire

hôte intermédiaire

syn. : hôte alternant ; hôte secondaire

hôte intermédiaire actif

hôte intermédiaire passif

hôte paraténique

syn. : hôte d'attente ; hôte de réenkystement

hôte primaire, syn. de hôte définitif

hôte de réenkystement, syn. de hôte paraténique

hôte secondaire, syn. de hôte intermédiaire

incapacité

incubation, syn. de période d'incubation

indicateur de santé

indice

infection

infestation

infirmité

invalidité

létalité

lutte contre la maladie

maladie contagieuse

maladie épidémique

maladie infectieuse

maladie transmissible

marqueur du risque

morbidité

morbidité totale, syn. peu usité de prévalence

mortalité

mortalité proportionnelle, syn. de

part de la mortalité due à une cause

(donnée)

pandémie

panzootie

part de la mortalité due à une cause donnée syn. : mortalité proportionnelle

pathogénicité

syn. : pouvoir pathogène

période d'incubation

syn. : incubation

control group; controls

handicap

host

definitive host, final host, primary host

intermediate host; intermediary host ; alternate host ; secondary hast

active intermediary host? passive intermediary host? paratenic host

incapacity ; disability

health status indicator

index

infection

infestation

infirmity

invalidity ; disablement

lethality; case fatality

disease control

contagious disease; contagion

epidemic disease

infectious disease

communicable disease

risk marker

morbidity; illness; morbid condition; sickness

mortality

pandemic : pandemia

panzootic

proportionate mortality ratio (abbr. PMR);

proportionate mortality; proportionate mortality

rate

pathogenecity; pathogenic potential

incubation period; incubative stage 
période de latence

période de prépatence

syn. : prépatence

phénomène de santé

population à haut risque

syn. : population à risque élevé

population à risque

population exposée (au risque)

population prédisposée

population vulnérable

pouvoir envahissant

pouvoir pathogène, syn. de pathogénicité

prépatence, syn. de

période de prépatence

prévalence

syn. : morbidité totale (peu usité);

fréquence globale (peu usité) ; fréquence (ambigu)

prévalence à un moment donné, syn. de prévalence ponctuelle

prévalence au cours d'une période donnée syn. : prévalence de période

prévalence instantanée, syn. de prévalence ponctuelle

prévalence de période, syn. de prévalence au cours d'une période donnée prévalence ponctuelle

syn. : prévalence à un moment donné ; prévalence instantanée

promotion

syn. : cohorte de mariage

proportion de cas dus au facteur, syn. de fraction étiologique du risque

randomisation, syn. de tirage au sort

rapport

recherche des cas

rendement

syn. : efficience

répartition des sujets au hasard, syn. de tirage au sort

risque

risque absolu

risque attribuable

risque relatif

sondage, syn. de enquête par sondage

sondage par grappes

surinfection

surveillance de la maladie

surveillance épidémiologique

taux

taux d'incidence

taux de mortalité

taux de mortalité par cause

taux de prévalence latency period; latent period

prepatent period

health problem (sens plus restreint)

high-risk population; population at risk (discutable)

population at risk

population at risk

population at risk; predisposed population

population at risk; vulnerable population

invasiveness

prevalence

period prevalence

point prevalence; prevalence at a point in time

ratio

case-finding

efficiency

risk

absolute risk

attributable risk

relative risk

cluster survey

1. cross infection

2. superinfection

surveillance of disease; surveillance of illness; monitoring of disease

epidemiological surveillance

rate

incidence rate

death rate; mortality rate

cause-specific death rate; cause-specific mortality prevalence rate 


\author{
témoin \\ utilité \\ vecteur \\ véhicule \\ virulence \\ vitesse de l'incidence
}

* Signe typographique proposé par les auteurs.

\section{LEXIQUE ANGLAIS-FRANÇAIS}

absolute risk

agent, syn. with causal agent

alternate host, syn. with

intermediate host

analysis

analytical study

attributable risk

attributable risk percent,

syn. with etiological fraction

birth cohort

blind trial

case

case-control study

case fatality, syn. with lethality

case-finding

causal agent

syn. : etiological agent; agent cause-specific death rate

syn. : cause-specific mortality

cluster survey

cohort

cohort study

syn. : incidence study

communicable disease

comprehensive survey

contagion

contagious disease

syn. : contagion

contamination

control

control group

syn. : controls

controlled intervention study,

syn. with controlled trial

controlled trial

cross infection

cross-sectional study

cross-sectional survey

death rate

syn. : mortality rate

definitive host

syn. : final host; primary host descriptive study

syn. : prevalence study control

efficacy

vector

vehicle

virulence

true incidence rate

risque absolu

analyse

étude analytique

risque attribuable

cohorte de naissance ; génération

essai à l'insu

cas

étude cas / témoins *

recherche des cas

agent causal; agent étiologique

taux de mortalité par cause

sondage par grappes

cohorte

étude de cohortes ; étude comparative des cohortes

maladie transmissible

enquête exhaustive

1. contagion

2. maladie contagieuse

3. contage

maladie contagieuse

contamination

témoin

groupe témoin

essai comparatif ; essai "contrôlé"

surinfection

étude transversale

enquête ponctuelie ; enquête transversale

taux de mortalité

hôte définitif ; hôte primaire

étude descriptive ; étude de prévalence 
directe contagion

syn. : immediate contagion

disability

syn. : incapacity

disablement, syn. with invalidity

disease control

double blind trial

effectiveness

efficacy

efficiency

endemic; endemia ; endemy

enzootic; enzootia ; enzooty

epidemic

epidemiological surveillance

epizootic; epizootia ; epizooty

eradication (of disease)

etiological agent, syn. with

causal agent

etiological factor

etiological fraction

Syn. : attributable risk percent; population attributable risk; population attributable risk proportion

experimentation

syn. : intervention study

experimenter

final host, syn. with

definitive host

frequency (of disease)

handicap

hazard rate, syn. with

incidence density

health problem

health status indicator

high-risk population

syn. : population at risk

$$
\text { (discutable) }
$$

host

illness, syn. with morbidity

immediate contagion, syn. with

direct contagion

impairment

incapacity, syn. with disability

incidence

incidence density

syn. : person-time incidence rate;

instantaneous incidence rate; hazard rate

incidence rate

incidence study, syn. with

cohort study

incubation period

syn. : incubative stage

index

indicator of health status,

see health status indicator

infection contagion directe

incapacité

lutte contre la maladie

essai à double insu; essai à double aveugle ; essai en double anonymat

efficacité

utilité

rendement ; efficience

endémie

enzootie

épidémie

surveillance épidémiologique

épizootie

éradication

facteur étiologique

fraction étiologique du risque; fraction attribuable, proportion de cas dus au facteur

expérimentation

expérimentateur

fréquence (de la maladie)

handicap

phénomène de santé (sens plus général)

indicateur de santé

population à haut risque ; population à risque élevé

hôte

déficience

incidence

densité de l'incidence

taux d'incidence

période d'incubation ; incubation

indice

infection 
infectious disease

infestation

infirmity

instantaneous incidence rate

syn. with incidence density

intermediary host, syn. with

intermediate host

intermediate host

syn. : alternate host; intermediary host ;

secondary host

intervention study, syn. with

experimentation

invalidity

syn. : disablement

invasiveness

latency period

syn. : latent period

lethality

syn. : case-fatality

life expectancy; life expectation

longitudinal study

mass screening

mediate contagion

monitoring of disease, syn. with

surveillance of disease

morbid condition, syn. with morbidity

morbidity

syn. : illness, morbid condition; sickness

mortality

mortality rate, syn. with death rate

multiphasic screening, syn. with multiple

screening?

multiple screening

syn. : multiphasic screening?

multi-step screening

observational epidemiology

pandemic ; pandemia

panzootic

paratenic host

pathogenic potential

syn. : pathogenecity

period prevalence

permanent survey

person-time incidence rate, syn. with

incidence density

PMR (abbr. for proportionate mortality ratio

point prevalence

syn. : prevalence at a point in time

population at risk

population attributable risk ;

population attributable risk proportion, syn.

with etiological fraction maladie infectieuse

infestation

infirmité

hôte intermédiaire ; hôte alternant ; hôte secondaire

invalidité

pouvoir envahissant

période de latence

létalité

espérance de vie

étude longitudinale

dépistage de masse

contagion indirecte

morbidité

mortalité

dépistage multiple

dépistage par examens successifs ; dépistage multiphasique?

épidémiologie d'observation

pandémie

panzootie

hôte paraténique; hôte d'attente; hôte de réenkystement

pathogénicité ; pouvoir pathogène

prévalence au cours d'une période donnée; prévalence de période

enquête permanente

prévalence ponctuelle; prévalence à un moment donné ; prévalence instantanée

1. population exposée (au risque)

2. population prédisposée

3. population vulnérable

4. population à haut risque; population à risque élevé (discutable) 
potential years of life lost

prepatent period

prevalence

prevalence at a point in time, syn. with point prevalence

prevalence rate

prevalence study, syn. with descriptive study

primary host, syn. with definitive host

proportionate mortality, syn. with proportionate mortality ratio

proportionate mortality ratio

(abbr. : PMR)

syn. : proportionate mortality proportionate mortality rate

prospective study

random allocation, syn. with

randomisation

randomisation (G.B.) ; randomization

(É.-U.)

syn. : random allocation

rate

ratio

receptive host, syn. with

susceptible host

retrospective study

risk

risk factor

risk marker

sample

sampling

sampling survey

screening

secondary host, syn. with

intermediate host

selective screening

sckness, syn. with morbidity

single blind trial

study

superinfection

surveillance of disease

syn. : surveillance of illness monitoring of disease

survey

susceptible host

syn. : receptive host

trial

true incidence rate

vector

vehicle

virulence années potentielles de vie perdues

(abrév. APVP)

période de prépatence; prépatence

prévalence, morbidité totale (peu usité) ; fréquence globale (peu usité) ; fréquence (ambigu)

taux de prévalence

part de la mortalité due à une cause (donnée) mortalité proportionnelle

étude prospective

tirage au sort ; répartition des sujets au hasard randomisation ; distribution aléatoire ; allocation aléatoire

taux

1. rapport

2. ratio

étude rétrospective

risque

facteur de risque; facteur du risque

marqueur du risque

échantillon

échantillonnage

enquête par sondage

dépistage

dépistage sélectif

essai à simple insu; essai à simple aveugle; essai en simple anonymat

étude

surinfection

surveillance de la maladie

enquête

hôte réceptif

essai

vitesse de l'incidence

vecteur

véhicule

virulence

Notes

* Le présent article est issu d'une recherche subventionnée par l'Office de la langue française du Québec.

+ Par opposition à une nouvelle venue, l'épidémiologie clinique. 


\section{Références}

1. MANUILA, L., A. MANUILA et M. NICOULIN (1977) : Petit dictionnaire médical, $2^{e}$ éd., Paris, Vevey, Masson, éd. Delta, p. 194.

2. JENICEK, Milos (1974) : Introduction à l'épidémiologie, Montréal, les Presses de l'Université de Montréal, p. 3.

3. HOGARTH, James (1977) : Vocabulaire de la santé publique, présentation par E.J. Aujaleu (et traduction française), Copenhague, OMS, Bureau régional de l'Europe, p. 93.

4. Dictionnaire de médecine Flammarion (1982) : Paris, Flammarion, Médecine-Sciences, p. 518.

5. MANUILA, A. et L., M. NICOLE et H. LAMBERT (1971) : Dictionnaire français de médecine et de biologie, t. II, Paris, Masson, p. 863.

6. RUMEAU-ROUQUETTE, Claude, Gérard BREART et René PADIEU, avec le concours de R. PRESSAT (1981) : Méthodes en épidémiologie, $2^{e}$ éd., Paris, Flammarion, Médecine-Sciences, pp. $167-168$.

7. MONNIER, J., J.-P. DESCHAMPS, J. FABRY, M. MANCIAUX, A.M. RAIMBAULT et coll. (1980) : Santé publique, santé de la communauté, Villeurbanne, Simep, p. 47.

8. JENICEK, Milos et Robert CLEROUX (1982) : Épidémiologie, St-Hyacinthe et Paris, Edisem et Maloine, p. 63.

9. RUMEAU-ROUQUETTE, Claude, op. cit., p. 167.

10. Ibid., p. 168.

11. MONNIER, J., op. cit., p. 42.

12. Le Petit Robert 1, Dictionnaire alphabétique et analogique de la langue française, par Paul ROBERT (1982) : Société du Nouveau Littré, Le Robert, p. 1548.

13. MANUILA, A. et L., M. NICOLE et H. LAMBERT (1972) : Dictionnaire français de médecine et de biologie, t. III, Paris, Masson, p. 500.

14. Dictionnaire de médecine, op. cit., p. 286.

15. JENICEK, Milos et Robert CLÉROUX, op. cit., p. 439.

16. MANUILA, A. et L., M. NICOLE et H. LAMBERT (1971) : op. cit, t. II, p. 65.

17. Dictionnaire de médecine Flammarion, op. cit., p. 279.

18. JENICEK, Milos et Robert CLÉROUX, loc. cit.

19. MANUILA, A. et L., M. NICOLE et H. LAMBERT (1972) : op. cit., t. III, p. 206.

20. BALTAZARD, M., cité par A. et L. MANUILA, M. NICOLE et H. LAMBERT (1970) : op. cit., t. 1, p. 152.

21. HOGARTH, James, op. cit., p. 59.

22. Dorland's Illustrated Medical Dictionary, 26th Ed. (1981) : Philadelphie, Saunders, p. 1183.

23. MANUILA, A., L. MANUILA et J.-C. SOURNIA (1982) : Dictionnaire français de médecine et de biologie, fasc. 20, supplément, Paris, Masson, p. 67.

24. RUMEAU-ROUQUETTE, Claude, op. cit., p. 33.

25. BERNARD, Paul-Marie et Claude LAPOINTE (1980): Épidémiologie et biostatistique : notions de base, Québec, Université Laval, p. 179.

26. JENICEK, Milos et Robert CLÉROUX, op. cit., p. 131.

27. MANUILA, A. et L., M. NICOLE et H. LAMBERT (1972) : op. cit., t. III, p. 705.

28. RUMEAU-ROUQUETTE, Claude, op. cit., p. 62.

29. Ibid., p. 13.

30. KENDALL, Sir Maurice G. and William R. BUCKLAND (1975) : A Dictionary of Statistical Terms, London, Longman Group Limited, p. 155.

31. SCHWARTZ, Daniel, Robert FLAMANT et Joseph LELLOUCH (1981) : $2^{\mathrm{e}}$ éd., l'Essai thérapeutique chez l'homme, Paris, Flammarion, Médecine-Sciences, p. 13.

32. MONNIER, J., op. cit., p. 58.

33. HARANT, Hervé et Alix DELAGE (1984) : l'Épidémiologie, (Coll. Que sais-je ?, n 607), Paris, Presses universitaires de France, p. 88.

34. Ibid., pp. 88-89.

35. MONNIER, J., op. cit., p. 57.

36. MANUILA, A., L. MANUILA et J.-C. SOURNIA (1982) : op. cit., (supplément), p. 150.

37. RUMEAU-ROQUETTE, Claude, op. cit., p. 9.

38. Le Petit Robert, cité dans Claude RUMEAU-ROUQUETTE, ibid., p. 211

39. MANUILA, A. et L., M. NICOLE et H. LAMBERT (1970) : op. cit., t. I, p. 629.

40. RUMEAU-ROUQUETTE, Claude, op. cit, p. 211.

41. Dictionnaire de médecine Flammarion, op. cit., p. 183.

42. MANUILA, A. et L., M. NICOLE et H. LAMBERT (I970) : , t. 1, loc. cit.

43. PRESSAT, Roland (1979) : Dictionnaire de démographie, Paris, PUF, p. 26 et p. 173.

44. La Gazette officielle du Québec du 25 août 1984.

45. MANUILA, A. et L., M. NICOLE et H. LAMBERT (1971) : op. cit., t. II, p. 718. 
46. Ibid., p. 710

47. Ibid., p. 733 .

48. Dictionnaire de médecine Flammarion, op. cit., p. 419.

49. MANUILA, A. et L., M. NICOLE et H. LAMBERT (1971) : t. II, op. cit., p. 514.

50. Encyclopédie Bordas, fasc. $n^{\circ} 61$, "Médecine $2 »$ (1976) : Paris, p. 1764.

51. MANUILA, A. et L., M. NICOLE et H. LAMBERT (1970) : t. 1, op. cit., p. 663.

52. MANUILA, A. et L., M. NICOLE et H. LAMBERT (1971) : t. II, op. cit., p. 513.

53. Dictionnaire de médecine Flammarion, op. cit., p. 390.

54. Ibid.

55. MANUILA, A. et L., M. NICOLE et H. LAMBERT (1972) : t. III, op. cit, p. 1103.

56. JENICEK, Milos et Robert CLÉROUX, op. cit., p. 247.

57. RUMEAU-ROUQUETTE, Claude, op. cit., p. 265.

58. Dictionnaire de médecine Flammarion, op. cit., p. 688.

59. LELLOUCH, J. (1976) : "Le risque, définitions et procédés de calcul ", in Revue d'épidémiologie et de santé publique, Paris, Masson, vol. 24, pp. 201-202.

60. JENICEK, Milos et Robert CLÉROUX, op. cit., p. 291.

61. Dictionnaire de médecine Flammarion, loc. cit.

62. MONNIER, J., op. cit., p. 355.

63. JENICEK, Milos et Robert CLÉROUX, op. cit., p. 155.

64. MONNIER, J., loc. cit.

65. Dictionnaire de médecine Flammarion, op. cit., p. 688.

66. RUMEAU-ROUQUETTE, Claude, op. cit., p. 274.

67. LEVIN, M.L., A.M. LILIENFELD (1973) : in American Journal of Epidemiology, 98-3, Baltimore, Johns Hopkins University School of Hygiene and Public Health, p. 231.

68. JENICEK, Milos et Robert CLÊROUX, loc. cit

69. Ibid., pp. 289-290.

70. Dictionnaire de médecine Flammarion, op. cit., p. 414.

71. Ibid., p. 366

72. JENICEK, Milos (1976) : Introduction à l'épidémiologie, St-Hyacinthe et Paris, Edisem et Maloine, pp. 388-389. 